\title{
ISOLATION AND CHARACTERIZATION OF PISANG TONGKAT LANGIT (Musa troglodytarum L.) ACS1 AND ACO1 GENE EXPRESSION DURING FRUIT RIPENING PROCESS
}

\author{
FENNY MARTHA DWIVANY ${ }^{1,2,3^{*}}$, KRISTI LENCI PATTY $^{1,4}$ and CINDY NOVIANTI ${ }^{1}$ \\ ${ }^{I}$ School of Life Sciences and Technology, Institut Teknologi Bandung, Bandung, Indonesia \\ ${ }_{2}^{2}$ Bioscience and Biotechnology Research Center, Institut Teknologi Bandung, Bandung, Indonesia \\ ${ }^{3}$ Bali International Research Center for Banana, Indonesia \\ ${ }^{4}$ Department of Agrotechnology, Faculty of Engineering and Agriculture, \\ Universitas Nani Bili Nusantara, Sorong, West Papua, Indonesia \\ *E-mail:fenny@sith.itb.ac.id
}

Accepted 25 April 2020, Published online 6 July 2020

\begin{abstract}
Pisang tongkat langit (Musa troglodytarum L.) is a banana cultivar originated from Eastern Indonesia. Previous studies showed that $A C S 1$ and $A C O 1$ are genes that play an important role in banana ethylene biosynthesis. However, characterization and analysis of $M$. troglodytarum ACS1 and ACO1 genes have not been reported. This study aims to isolate and characterize the $A C S 1$ and $A C O 1$ gene expression profile during fruit ripening process. Gene fragment isolation was performed using PCR based method. Results showed that the isolated genomic fragments of $A C S 1$ was 400 bp and $A C O 1$ was 500 bp. cDNA fragments of $A C S 1$ was $200 \mathrm{bp}$ and $A C O 1$ was $300 \mathrm{bp}$. Analysis of genomic fragments using BLAST NCBI showed that ACS1 had a high homology with 1818 bp ACS1 gene of Musa acuminata AAA Group (GenBank: GQ396304.1), while ACO1 gene had the highest homology with 1593 bp ACO1 gene of M. acuminata AAA Group (GenBank: AJ223232.1). ACS1 genomic fragments of M. troglodytarum had one intron and two exons, while ACO1 had two introns and three exons. Homology analysis showed that gene fragment of $A C S 1$ and $A C O 1$ of $M$. troglodytarum had conserved region similar to M. acuminata.
\end{abstract}

Key words: Pisang tongkat langit, Musa troglodytarum, $A C S 1, A C O 1$, fruit ripening

\section{INTRODUCTION}

Bananas are the world's number four food commodity after rice, wheat, and corn. In 2013, world banana productivity reached 105.96 million tons, however Indonesia ranked sixth bananaproducing countries of total world production (FAOSTAT, 2016). One of local banana cultivars in Indonesia that has economic potential is pisang tongkat langit (Musa troglodytarum L.). Pisang tongkat langit grows naturally only in eastern Indonesia, namely in Maluku to the territory of French Polynesia (Lim, 2012). Pisang tongkat langit has unique characteristics compared to bananas in general, i.e. has bunches that grow upwards, purple sap, and are generally resistant to pests and diseases. People in Maluku have developed pisang tongkat langit as an alternative food and used them as a raw

\footnotetext{
* To whom correspondence should be addressed.
}

material for medicine (Cheesman, 1949; Satuhu \& Supriyadi, 2005; Ploetz et al., 2007).

Pisang tongkat langit is an interspecific hybrid, which is included in Australimusa banana group (Ploetz et al., 2007). However, the genetic data on this banana group is still very limited. Meanwhile, molecular studies on the ripening process of pisang tongkat langit has not been reported.

Bananas are included in climacteric fruit, where its ripening stages are associated with an increased rate of respiration and ethylene production. Ripening process of climacteric fruit is regulated by three consecutive enzymes i.e. SAM synthetase (S-adenosylmethionine synthetase), ACC synthase (ACS) and ACC oxidase (ACO). SAM synthetase catalyzes methionine to SAM (Sadenosylmethionine). SAM becomes MTA (5methylthioadenine), which enters into methionine cycle and is catalyzed by ACC synthase (ACS to become a sulfur atom and 1-aminocyclopropane-1- 
carboxylic acid (ACC)). Furthermore, in the presence of oxygen, ACC oxidase (ACO) will catalyze ACC into ethylene and $\mathrm{CO}_{2}$ (Yang \& Hoffmann, 1984). ACC synthase enzyme is encoded by $A C S$ gene while ACC oxidase is encoded by $A C O$ gene. $A C S$ and $A C O$ genes are gene families, whose expression is regulated by many different signals such as environment, fruit development, and number of plant hormones (Karmawan et al., 2009; Dwivany et al., 2016). Recently, it has been known that ACS1 and $A C O 1$ genes have an important role in fruit ripening. Research on both genes is widely performed in Musa acuminata AAA Group but has never been done in pisang tongkat langit (Musa troglodytarum L.). Therefore, this research is focused on the characterization and analysis of pisang tongkat langit $A C S 1$ and $A C O 1$ genes as well as to determine whether the process of fruit ripening in pisang tongkat langit is correlated with genes that play a role in fruit ripening. The results from this study can be used for future postharvest management of pisang tongkat langit.

\section{MATERIALS AND METHODS}

\section{Materials}

Pisang tongkat langit (Musa troglodytarum L.) used for this study were obtained from Ullath Village, Saparua Island, Central Maluku, Maluku Province, Indonesia. Pisang tongkat langit were transported carefully to avoid any physical damage.

\section{Isolation and analysis of $A C S 1$ and $A C O 1$ genes}

Isolation of DNA fragments from fruit pulp was done using the method by Doyle and Doyle (1990) which has been modified by Lodhi et al. (1994). Primers used in PCR analysis were ACS1, ACO1, and GAPDH (housekeeping gene) of Musa acuminata AAA Group which has been optimized by Karmawan et al. (2009) and Handayani and Dwivany (2014) (Table 1). The DNA fragments were amplified using GoTaq ${ }^{\circledR}$ Green Master Mix reagents from Promega (Catalog No. M7122). DNA fragments were sequenced using sequencing services from MACROGEN and the results were identified by BLASTN (www.ncbi.nlm.nih.gov/BLAST).
Isolation of RNA from banana fruit pulp on day 1 of fruit ripening was done using Cordeiro et al. (2008) method with modification, then the RNA fragments were used as templates for cDNA synthesis using an iScript ${ }^{\mathrm{TM}}$ cDNA Synthesis (Biorad, Catalog No. 170-8890). The cDNA was amplified using $A C S 1, A C O 1$, and $G A P D H$ primers listed in Table 1. The cDNA fragments were sequenced using sequencing services from MACROGEN. Sequencing Results were identified by BLASTN (www.ncbi.nlm.nih.gov/BLAST), then aligned using Clustal Omega - Multiple Sequence Aligment (https:/www.ebi.ac.uk/Tools/ $\mathrm{msa} /$ clustalo/) to compare both $A C S 1$ and $A C O 1$ gene structures.

In addition, BLASTX (www.ncbi.nlm.nih.gov/ BLAST) analysis was used to analyze structures of ACS1 and ACO1 proteins. Gene alignment analysis was done using clustalW of BIOEDIT and the MEGA 5.2 was used to construct the phylogenetic tree.

\section{RESULTS AND DISCUSSION}

\section{Gene isolation and characterization}

Pisang tongkat langit DNA amplification was performed using ACS1, ACO1 and GAPDH primers, which were designed from Musa acuminata AAA Group. Results showed that GAPDH gene fragment was $250 \mathrm{bp}, A C S 1$ was $400 \mathrm{bp}$ and $A C O 1$ was 500 bp (Figure 1A). Identification of $A C S 1$ and $A C O 1$ genes using BLASTN showed that the ACS1 gene had $93 \%$ similarity with ACS1 of Musa acuminata AAA Group (access code GenBank: GQ396304.1), while $A C O 1$ had $96 \%$ similarity with $A C O 1$ of Musa acuminata AAA Group (access code Gen Bank: AJ223232. 1). Full-length ACS1 gene of Musa acuminata AAA Group was 1,818 bp in size (Karmawan et al., 2009), while full-length ACO1 of Musa acuminata AAA Group was 1,593 bp in size (Chen et al., 1999).

Pisang tongkat langit cDNA amplification was performed using same $A C S 1, A C O 1$ and $G A P D H$ primers for amplifying the pisang tongkat langit DNA. Results showed that GAPDH cDNA fragment was around $250 \mathrm{bp}, A C S 1$ was $200 \mathrm{bp}$, and $A C O 1$

Table 1. Primer sequences used in PCR analysis

\begin{tabular}{llcl}
\hline Primer & Oligonucleotide sequence $\left(5^{\prime} \rightarrow 3^{\prime}\right)$ & Size $(\mathrm{bp})$ & Reference \\
\hline MaGAPDH RT-F & TCAACGACCCCTTCATCAC & 250 & Karmawan et al., 2009 \\
MaGAPDH RT-R & AGCAGCCTTGTCCTTGTCA & & \\
MaACS1 RT-F & CCGAGACTGGATGAAGAAGAA & 172 & Karmawan et al., 2009 \\
MaACS-1 RT-R & GTCTGGGTCAAATCTGGCTC & & \\
MaACO1 RT-F & CGAGATGCTTGCGAGAAATGG & 240 & Handayani \& Dwivany, 2014 \\
MaACO1 RT-R & TGCAGCAAATTCCTTCATCGC & & \\
\hline
\end{tabular}




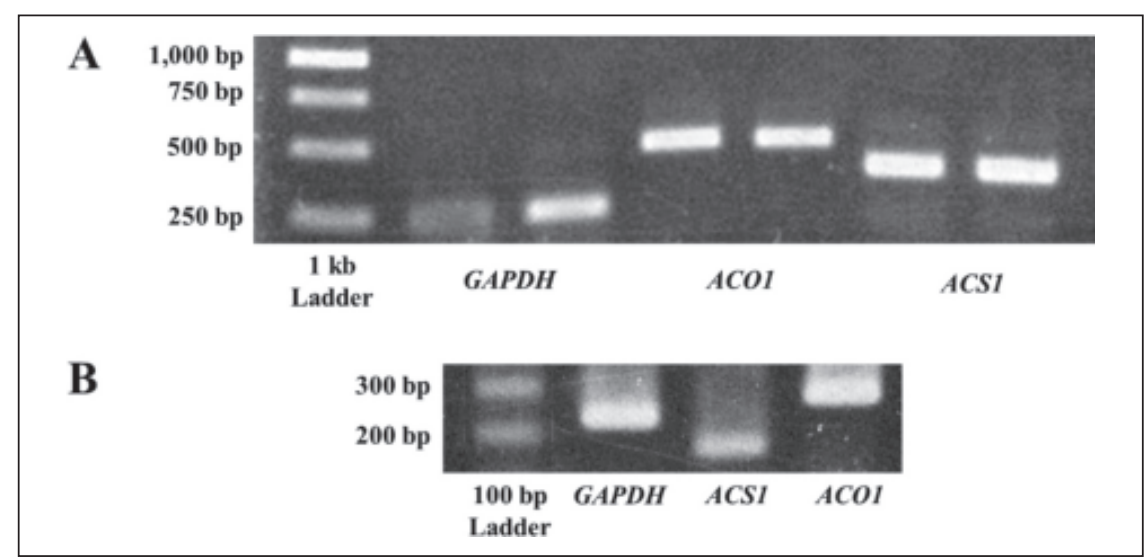

Fig. 1. Electropherogram of $A C S 1, A C O 1$ and $G A P D H$ gene fragments. (A) Gene fragments amplification using genomic DNA of pisang tongkat langit (Musa troglodytarum L.). (B) Gene fragments amplification using cDNA of pisang tongkat langit (Musa troglodytarum L.) pulp on day 1 of fruit ripening.

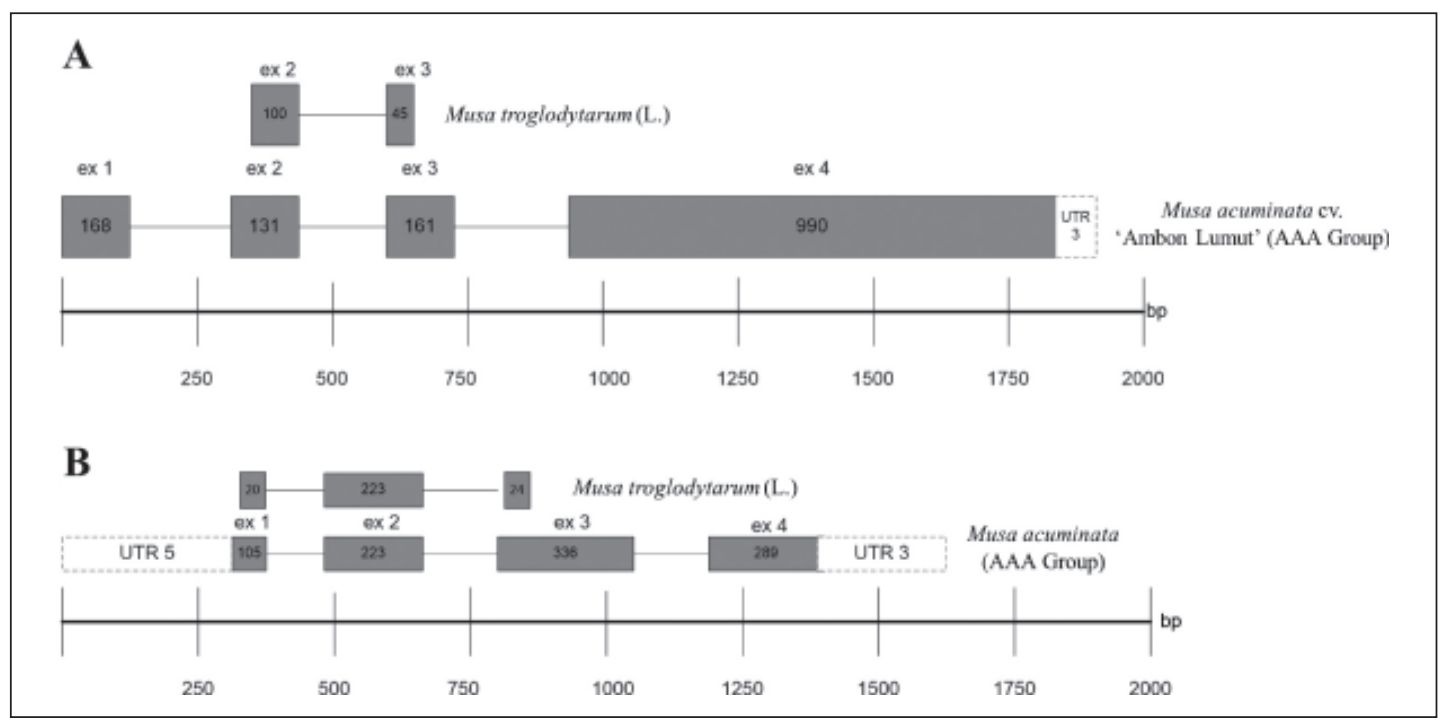

Fig. 2. Pisang tongkat langit (Musa troglodytarum L.) and Musa acuminata AAA Group ACSI (A) and ACOI (B) gene structure.

was $300 \mathrm{bp}$ (Figure 1B). Identification of $A C S 1$ and $A C O 1$ cDNA using BLASTN showed that the ACS1 cDNA fragment had $97 \%$ similarity with ACS1 of Musa acuminata AAA Group mRNA (access code GenBank: Y15739.1), while $A C O 1$ cDNA fragment had 92\% similarity with ACO1 of Musa acuminata AAA Group mRNA (access code Gen Bank: EU131109.1). According to Choudhury et al. (2008), M. acuminata (AAA Group, Subgroup Cavendish) has 1,477 bp of full-length $A C S 1$ cDNA sequence and 957 bp of full-length $A C O 1$ cDNA sequence. Moreover, Dwivany et al. (2016) reported that both genes were also expressed during fruit ripening in "Raja Bulu" banana while other MaACS gene (MaACS2) was expressed in leaf and root of fungi-infected banana tissue culture (Handayani \& Dwivany, 2014).

Schematic structure of the $A C S 1$ and $A C O 1$ gene fragments of pisang tongkat langit (Musa troglodytarum L.) compared with Musa acuminata AAA Group can be seen in Figure 2 (the alignment results could be seen in Supplemental Data 1 and 2). The exon and intron of $A C S 1$ and $A C O 1$ were found using alignment tool of Clustal Omega, the results of which can be seen in Supplemental Data 3 and 4.

\section{Sequence alignment and phylogenetic analysis of $A C S 1$ and $A C O 1$ genes}

Protein sequence alignment analysis of $A C S 1$ (Figure 3) showed amino acid sequence differences between Musa acuminata AAA Group and pisang tongkat langit (Musa troglodytarum L). Results of BLASTX (Figure 3) showed that protein sequences obtained from both bananas have conserved sequences 'FQDYHGLP' as mentioned by Karmawan et al. (2009). However, ACS1 protein sequences of pisang tongkat langit have three 


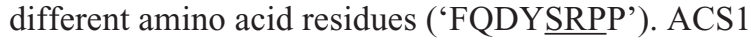
protein in bananas forms homodimers and forms a complex with the cofactor PLP (pyridoxal 5 'phosphate), amino acid residues Ser-Leu-Ser-LysSer-Lys-Asp-Leu-Val-Gly-Pro-Gly-Phe-Arg-Val-Gly (sequence no. 276-289) were mentioned as a site to bind to aminotransferase class-1 pyridoxalphosphate (Choudhury et al., 2009). However, further study to isolate full-length sequence of ACS1 protein is needed.

Phylogenetic analysis showed four main groups based on branching: Group 1 consisted of
Musa acuminata AAA Group and their cultivars. Group 2 consisted of pisang tongkat langit (Musa troglodytarum L.), Musa acuminata AAA Group, Musa acuminata AAA Group cv. 'Ambon Lumut', Musa acuminata AAA Group clone GMACS12 and GMACS clone-9. Group 3 consisted of Arabidopsis thaliana derived from dicotyledonous plant group and group 4 consisted Saccharum officinarum representing the group of monocots plants and Musa acuminata AAA Group GMACS clone-1 (Figure 4).

Protein alignment of both pisang tongkat langit and Musa acuminata AAA Group (GenBank

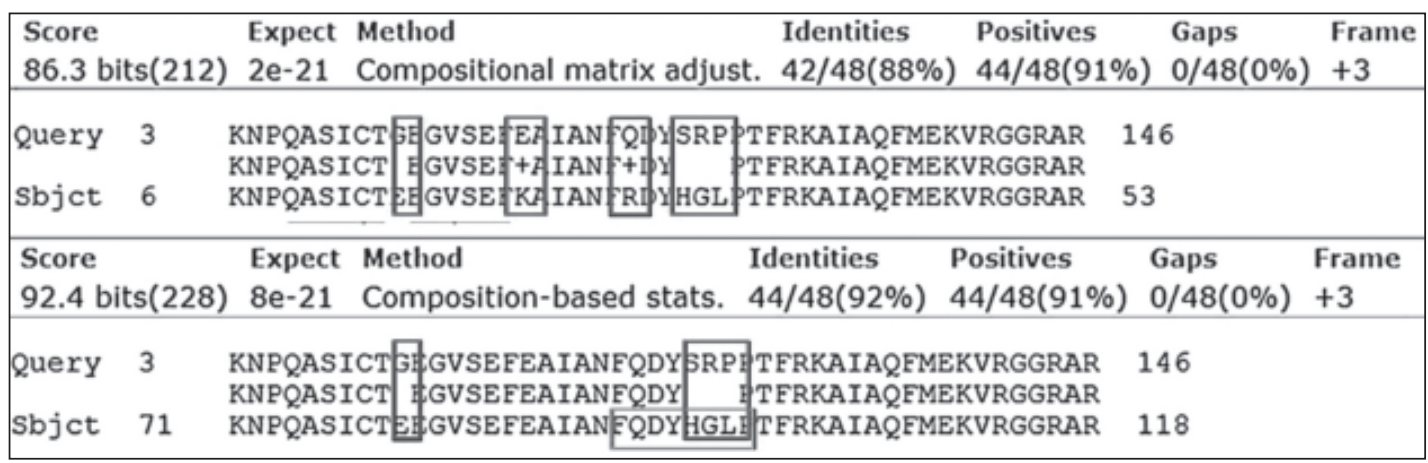

Fig. 3. The ACS1 protein alignment. Black box shows sequence differences. Grey box shows conserved area for ACS. Query: pisang tongkat langit (Musa Troglodytarum L.) ACS1, Subject: Musa acuminata AAA Group ACS1 (access code GenBank: ACV30004.1; CAA75749.1).

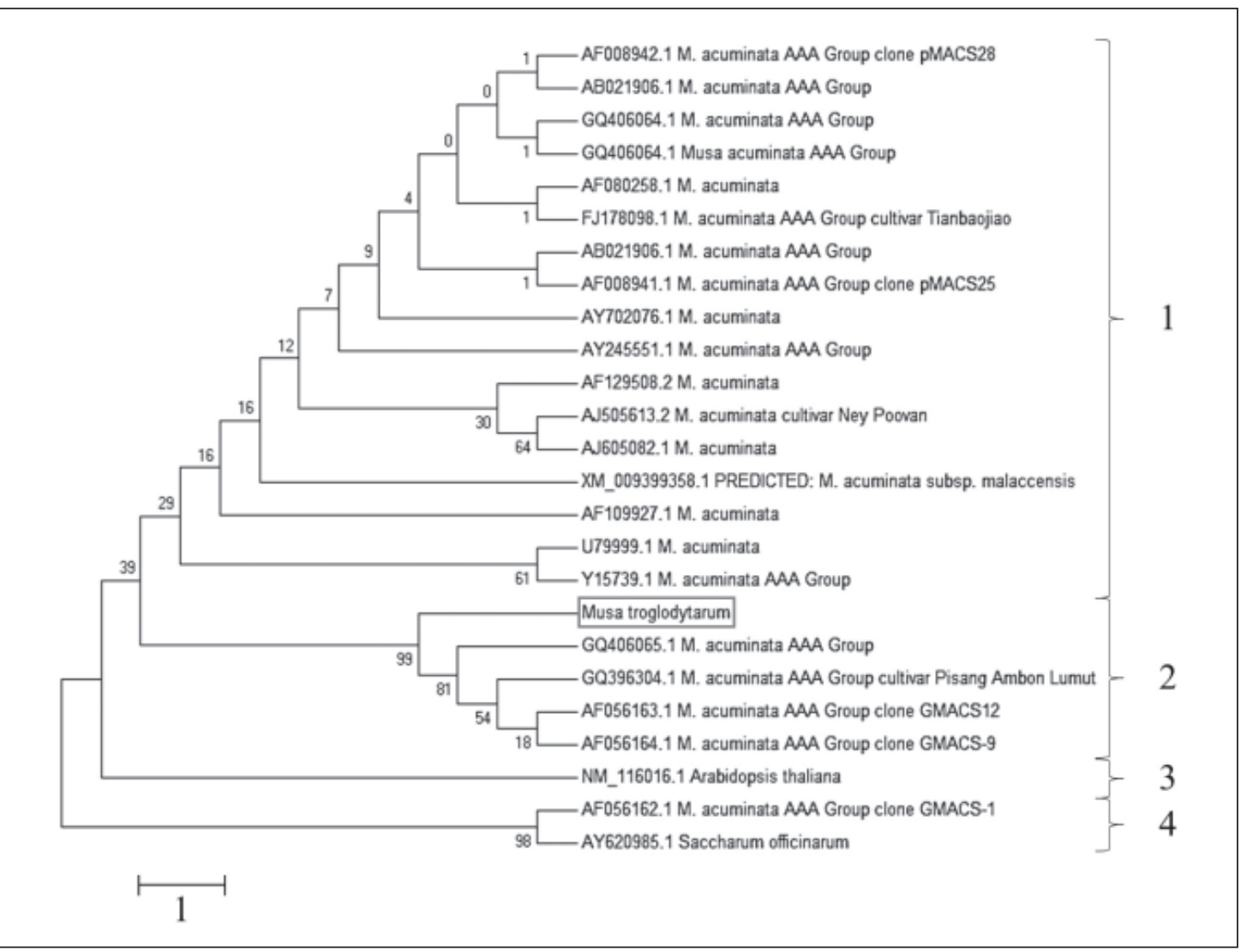

Fig. 4. Phylogenetic tree based on nucleotide sequence alignment of ACS1 from Musa acuminata AAA Group, Musa troglodytarum, Arabidopsis thaliana and Saccharum officinarum using bootstrap consensus neighbor-joining. 
access code: CAA11200.1) ACO1 are shown in Figure 5 . There are only three amino acid sequence differences between Musa acuminata AAA Group and pisang tongkat langit on sequence 42,58 , and 60. It has been reported previously that ACO enzyme family is highly conserved (Hubert \& Mbeguie-AMbeguie, 2012). ACC oxidase (ACO) is a member of superfamily group of non-heme-iron that utilizes $\mathrm{Fe}$ (II) as a co-factor and 2-oxoglutarat (20G) as cosubstrat (John, 1991; Bidonde et al., 1998).

Phylogenetic analysis showed the presence of two main groups based on branching: Group 1 consisted of Musa acuminata AAA Group, their cultivars and pisang tongkat langit (Musa troglodytarum L.), Group 2 consisted of Musa ABB Group, M. indica which represents dicot plant and Oryza sativa representing the monocot plant (Figure 6).

Results from this study showed that ACS1 and $A C O 1$ gene fragments were successfully isolated from pisang tongkat langit (Musa troglodytarum L.), which also having high similarity to Musa acuminata. Both genes were also expressed during fruit ripening process, which was indicated by cDNA amplification result (Figure 1B).

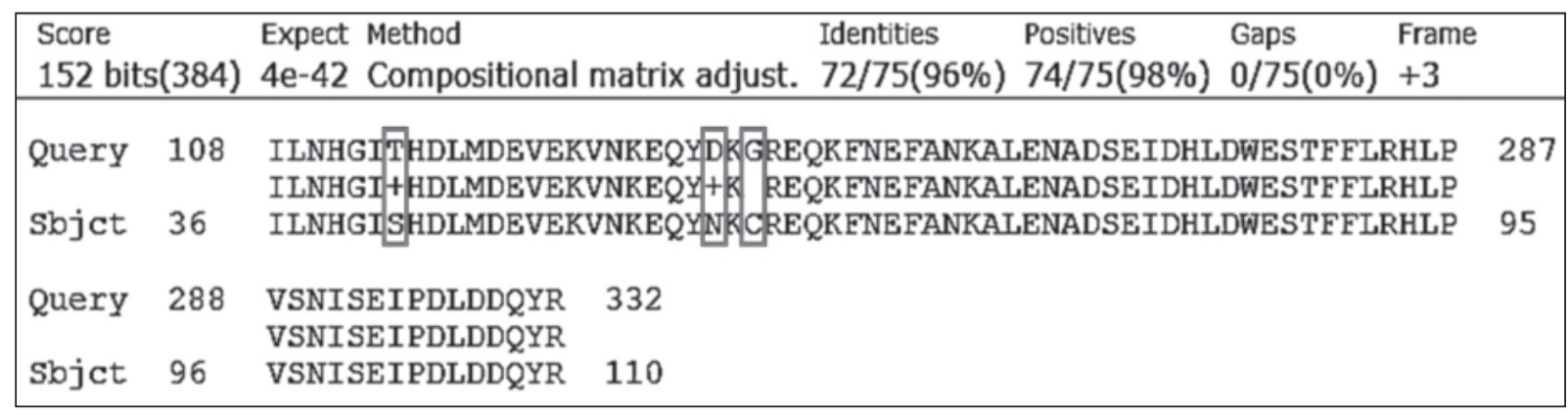

Fig. 5. The ACO1 protein alignment. Black box shows sequences differences. Query: pisang tongkat langit (Musa troglodytarum L.) ACO1, Subject: Musa acuminata AAA Group ACO1 (GenBank access code: CAA11200.1).

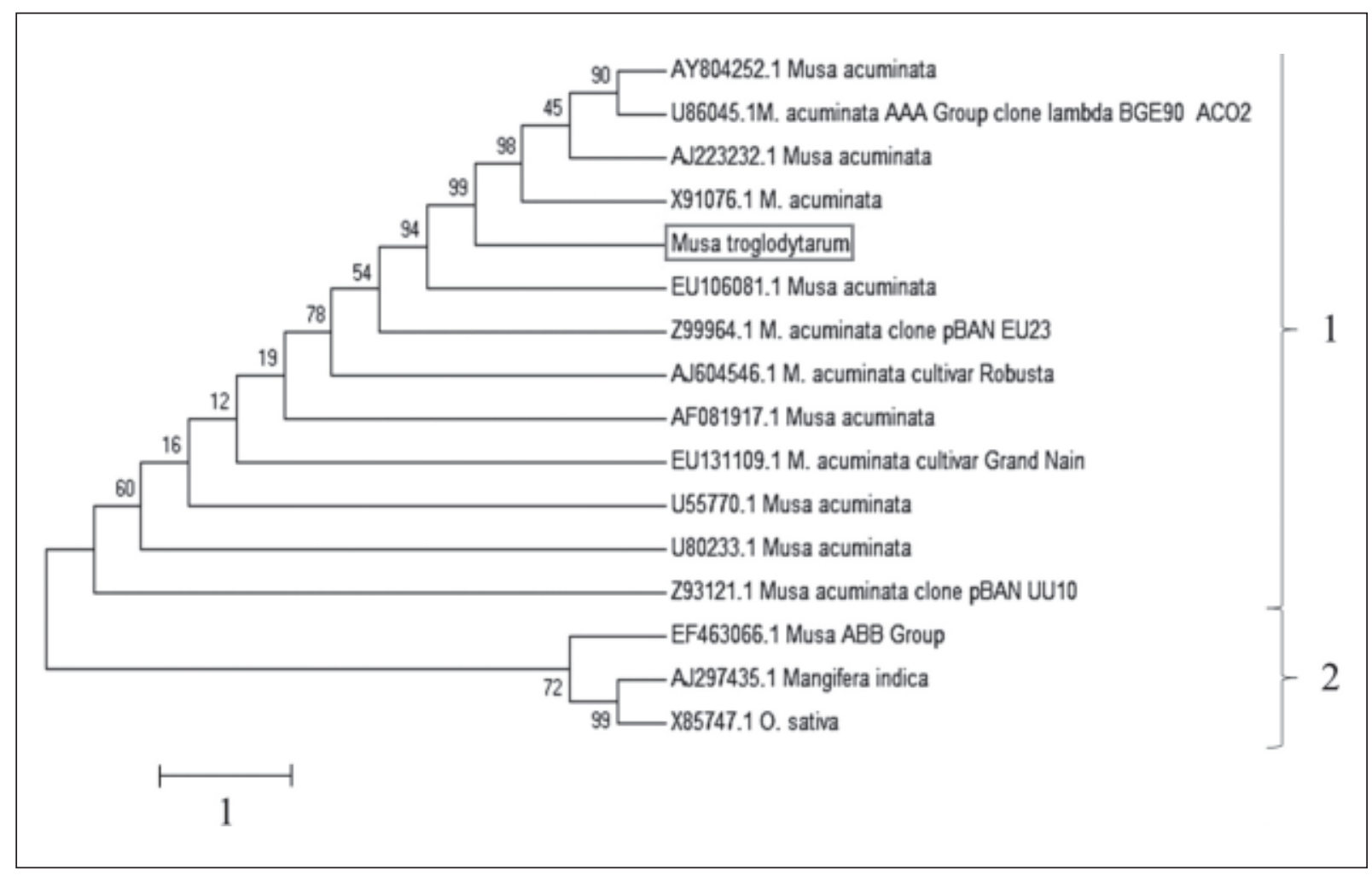

Fig. 6. Phylogenetic tree based on nucleotide sequence alignment of ACO1 from Musa acuminata AAA Group, pisang tongkat langit (Musa troglodytarum L), M. indica, Musa ABB Group and Oryza sativa using Bootstrap consensus neighborjoining. 


\section{ACKNOWLEDGEMENTS}

Author would like to thank Institut Teknologi Bandung, Indonesia and Ministry of Research, Technology and Higher Education, Indonesia for supporting the research.

\section{REFERENCES}

Bidonde, S., Ferrer, M.A. \& Zegzouti, H. 1998. Expression and characterization of three tomato 1-aminocyclopropane-1-carboxylate oxidase cDNAs in yeast. European Journal of Biochemistry, 253(1): 20-26. [doi:10.1046/ j.1432-1327.1998.2530020.x]

Cheesman, E.E. 1949. Classification of the bananas: Critical notes on species: Musa fehi. Kew Bulletin, 4(4): 445-449. [doi:10.2307/4109051]

Chen, Y.M., Lu, B.W., Hagenbeek, D., Li, N. \& Yang, S.F. 1999. A nucleotide sequence of Musa accuminata cv. Cavendish Grand Nain (AAA) encoding an ethylene inducible 1Aminocyclopropane-1-Carboxylate Synthase (accession no. AJ223232) (PGR 99-062). Plant Physiology, 120: 340.

Choudhury, S.R., Roy, S. \& Sengupta, D.N. 2008. Characterization of transcriptional profiles of $M A-A C S 1$ and MA-ACO1 genes in response toethylene, auxin, wounding, cold and different photoperiods during ripening in banana fruit. Journal of Plant Physiology, 165: 1865-1878. [doi:10.1016/j.jplph.2008.04.012]

Choudhury, S.R., Roy, S. \& Sengupta, D.N. 2009. $M A-A C S 1$ : A key operator in ethylene biosynthesis in banana - its role and regulation during fruit ripening. ISHS Acta Horticulturae, 897. [doi: 10.177660/ActaHortic.2011.897.20]

Cordeiro, M.C.R., Silva, M.S., Oliveira-Filho, E.C., de Miranda, Z.J.G., Aquino, F.G., Fragoso, R.R., Almeida, J. \& Andrade, L.R.M. 2008. Optimization of a method of total RNA extraction from Brazilian native plants rich in polyphenols and polysaccharides. In: IX Simposio Nacional Cerrado. October 12-17, 2008, ParlaMundi, Brazil.

Doyle, J.J \& Doyle, J.L. 1990. Isolation of plant DNA from fresh tissue. Focus, 12: 13-15.

Dwivany, F.M., Hermawaty, D. \& Esyanti, R.R. 2016. 'Raja Bulu' banana MaACS1 and $\mathrm{MaACO1}$ gene expression during postharvest storage. Acta Hortic, 1120: 111-114. [doi: 10.17660/ActaHortic.2016.1120.16]
FAOSTAT. Production and Trade (2016). FAO, http://faostat.fao.org/. Accessed on January 2016.

Handayani, R.U. \& Dwivany, F.M. 2014 . Analysis of MaACS2, a stress-inducible ACC Synthase Gene in Musa acuminata AAA Group Cultivar Pisang Ambon. Journal of Mathematical and Fundamental Sciences, 46(2): 194-203. [doi: 10.5614/j.math.fund.sci.2014.46.2.8]

Hubert, O. \& Mbéguié-A-Mbéguié, D. 2012. Expression Patterns of Ethylene Biosynthesis Genes from Bananas During Fruit Ripening and in Relationship with Finger Drop. AoB Plants, pls041. [doi:10.1093/aobpla/pls041]

John, P. 1991. How plant molecular biologists revealed a surprising relationship between two enzymes, which took an enzyme out of a membrane, where it was not located and put it into the soluble phase where it could be studied. Plant Molecular Biology Reporter, 9(3): $192-$ 194. [doi:10.1007/BF02672067]

Karmawan, L.U., Suhandono, S. \& Dwivany, F.M. 2009. Isolation of $M A-A C S$ gene family and ekspression study of MA-ACS1 gene in Musa acuminata Cultivar Pisang Ambon Lumut. Hayati Journal of Biosciences, 16(1): 35-39. [doi: 10.4308/hjb.16.1.35]

Lim, K.T. 2012. Edible medicinal and nonmedicinal plants. Vol. 3: Fruits. Springer Science + Business Media B.V, Dordrecht.

Lodhi, M.A., Guang-Ning Ye, Norman, F.W. \& Bruce, I.R. 1994. A simple and efficient method for DNA extraction from grapevine cultivars, vitis species and Ampelopsis. Plant Molecular Biology Reporter, 12(1): 6-13. [https://doi.org/ 10.1007/BF02668658]

Ploetz, R.C., Kepler, A.K., Daniells, J. \& Nelson, S.C. 2007. Banana and Plantain-an overview with emphasis on Pasific Island Cultivars. Species Profiles for Pasific Island. Agroforestry, 1: 1-27.

Satuhu, S. \& Supriyadi, A. 2005. Pisang: Budi daya, pengolahan, dan prospek dasar. Penebar Swadaya, Jakarta. (Bahasa Indonesia)

Yang, S.F. \& Hoffman, N.E. 1984. Ethylene biosysnthesis and its regulation in higher plants. Annual Review of Plant Physiology, 35: 155-189. [https://doi.org/10.1146/annurev. pp.35.060184.001103] 


\section{SUPPLEMENTAL DATA}

Supplemental Data 1. Alignment result of Musa acuminata cv. 'Ambon Lumut' AAA Group and pisang tongkat langit (Musa troglodytarum L.) ACS1 gene sequence. Starred sequences shows similar sequences (considered as conserved sequences).

\section{ACS1 M. acuminata} ACS1_M.troglodytarum

ACS1 M.acuminata ACS1_M.troglodytarum

ACS1 M.acuminata ACS1_M.troglodytarum

ACS1 M.acuminata ACS1_M.troglodytarum

ACS1_M. acuminata ACS1_M.troglodytarum

ACS1 M.acuminata ACS1_M.troglodytarum

ACS1_M.acuminata ACS1_M.troglodytarum

ACS1 M.acuminata ACS1_M.troglodytarum

ACS1 M.acuminata ACS1_M.troglodytarum

ACS1_M.acuminata ACS1_M.troglodytarum

ACS1_M.acuminata ACS1_M.troglodytarum

ACs1 M.acuminata ACS1_M.troglodytarum

ACS1 M.acuminata ACS1_M.troglodytarum

ACS1 M.acuminata ACS1_M.troglodytarum

ACS1 M.acuminata ACS1_M.troglodytarum
TACGGCGAGGAGCACCCAAATCAGCAGATCCTCTCTCGGATCGCGACCAACGACGGCCAT 60

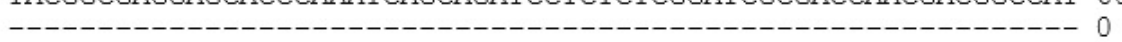

GGCGAGAACTCCTCCTACTTCGATGGGTGGAAGGCCTACGAGAAGGATCCTTTCCACCTC 120 ----------------------------------------------------------- 0

ACCGACAACCCCACGGGGGTCATCCAAATGGGACTCGCAGAAAACCAGGTTAGAGTTCCT 180

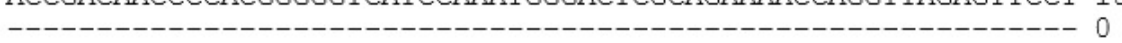

TCATGGTGATGATTAATCGCACATGCCTTCCGTCAATTGCCACTCCCTGCGGTTGCTAAT 240 --------------------------------------------------------- 0

CTAATCTGTATGTGGGTTTGGGTCTTTCTTTCCTCAGCTTTCCCTCGACTTGATCCGAG 300

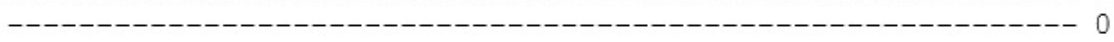

ACTGGATGAAGAAGAACCCACAGGCTTCGATCTGCACCGAAGAAGGGGTCTCAGAGTTCA 360 -------AGAAGAACCCACAGGCTTCGATCTGCACCGGGGAAGGGGTCTCAGAGTTCG 51

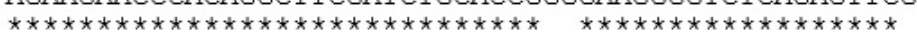

AAGCAATTGCCAACTTTCAGGACTATCATGGCCTCCCAGCCTTCCGAAAGGTAATGATTT 420 AAGCAATTGCCAACTTTCAGGACTATTCACGGCCTCCCACCTTCCGAAAGGTACTGATTT 111 $\star * * * * * * * * * * * * * * * * * * * * * * * * * \quad * * \quad * * \quad * * * * * * * * * * * * * * * * * * * *$

CAACCCAAAACGCAGCGCTGCAGCTGCTTGTCCTCACTGTCCAAGTAGCTACATACGTCC 480 CAACCCGAAACGCAGAGCTGCAGCTGCTTGTCCTCACTGTCCAAGTAGCTACATACGTCC 171 $* * * * * * \quad * * * * * * * * \quad * * * * * * * * * * * * * * * * * * * * * * * * * * * * * * * * * * * * * * * * * * * *$

AATATGATAAAGCTGGGACTGACAGCCACTTACGGCCCGAGCCCTGCCTGCTCACCCTGG 540 AATACGATAAAGCTGGGACTGACAGCTACCTACGGCCTGAGACCTACCTGCTCACGCTGG 231 $\star * * * * * * * * * * * * * * * * * * * * * * * * * * * * * * * * * * * * * * * * * * * * * * * * * * * *$

ATAAGGGATAAGCTAATGATGGTGTGATTTGCTGACACGCGCAGGCCATCGCCCAGTTCA 600 AT--GGGATAAGCTAATGATGATGTGATTTGCCGACACGCGCAGGCCATCGCCCAGTTCA 289 $\star * * \quad * * * * * * * * * * * * * * * * * \quad * * * * * * * * * * \quad * * * * * * * * * * * * * * * * * * * * * * * * * * *$

TGGAGAAGGTGAGAGGGGGACGAGCCAGATTTGACCCAGACCGCATCGTGATGAGCGGTG 660

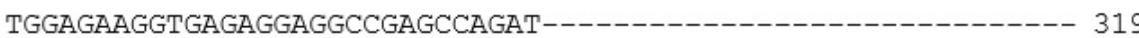
$\star * \star * * * * * * * * * * * * * * * * * * * * * * * * *$

GAGCCACCGGCGCTCAGGAAACCATCGCCTTTTGCCTGGCTGATCCTGGCGAGGCCTTCT 720

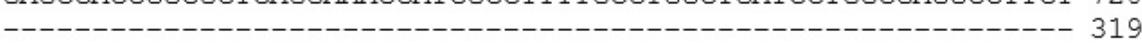
TGATTCCAACGCCATATTATCCGGGGTAAGTATTTAGGTGTACTAATCTACCGAGTTCTT 780

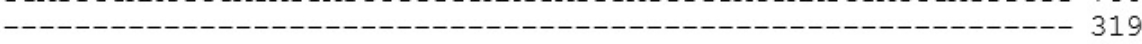
TATCCGGCAGAGGATCTAATGGCATCTGCATGGTTTCCAGATTCGATCGAGACTTCAGGT 840 ---------------------------------------------------------- 319 GGAGGACAGGAGTTCAGCTCCTCCCCATTCACTGCCACAGTTCCAACAAGTTCAAGATCA 900 
ACS1 M.acuminata ACS1_M.troglodytarum

ACS1 M.acuminata ACS1_M.troglodytarum

ACS1 M.acuminata ACS1_M.troglodytarum

ACS1 M.acuminata ACS1_M.troglodytarum

ACS1 M.acuminata ACS1_M.troglodytarum

ACS1 M.acuminata ACS1_M.troglodytarum

ACS1 M.acuminata ACS1 M. troglodytarum

ACS1 M.acuminata

ACS1_M.troglodytarum

ACS1 M.acuminata

ACS1_M.troglodytarum

ACS1 M.acuminata

ACS1-M.troglodytarum

ACS1 M.acuminata

ACS1_M.troglodytarum

ACS1 M.acuminata

ACS1_M.troglodytarum

ACS1 M.acuminata

ACS1_M.troglodytarum

ACS1 M.acuminata

ACS1-M.troglodytarum

ACS1 M.acuminata

ACS1 $^{-}$M.troglodytarum

ACS1 M.acuminata

ACS1_M. troglodytarum

ACS1 M.acuminata

ACS1 M. troglodytarum
CCCAAGCCGCACTGGAGACTGCTTACAGGAAGGCTCGAAACTCACACATTAGAGTCAAAG 960

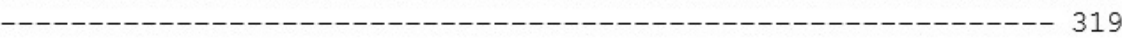

GAATACTGGTGACCAACCCATCGAACCCTCTGGGCACAACCATGGACAGAGAGACGCTGA 1020

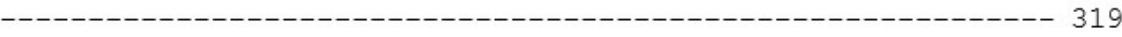

GAACCCTAGTCAGCTTCGTCAACGAGAAAAGGATGCACTTGGTGTGCGACGAGATCTTCT 1080

CCGGAACCGTCTTCGACAAGCCGAGTTACGTGAGCGTCTCCGAGGTGATCGAAGACGAGC 1140

CCTACTGCGACAGGGATCTGATTCACATCGCCTACAGCCTCTCCAAGGACCTGGGCGTCC 1200

CTGGCTTCCGCGTCGGCGTCATATACTCCTACAACGACGCCGTGGTCAGCTGCGCGAGGA 1260

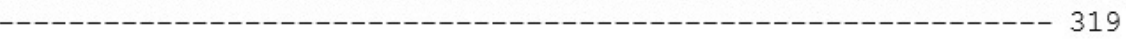

AGATGTCGAGCTTTGGACTGGTCTCGTCGCAGACGCAGCACCTGCTCGCTTCCATGTTGG 1320

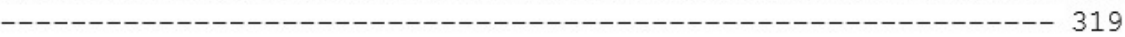

GAGACGAGGAGTTCACCACGAGTTTCTTAGCGACGAGCCGGACGAGGTTGTGCGGGCGGC 1380

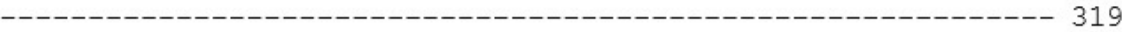

GAGACGAGGAGTTCACCACGAGTTTCTTAGCGACGAGCCGGACGAGGTTGTGCGGGCGGC 1380

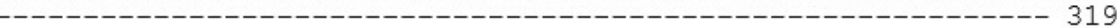

GCAGGGTCTTTACGGACGGCCTCAAGCGAGTCGGGATTCATTGCTTGGACGGCAACGCGG 1440

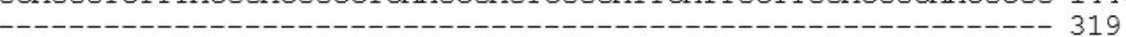

GGCTGTTCTGCTGGATGGACTTGAGGCCGTTGCTGAAGGAAGCGACGGTGGAGGCGGAGC 1500

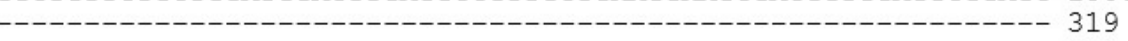

TCCGGCTGTGGCGGGTGATCATCAACGACGTGAAGCTCAACATCTCGCCGGGGTCGTCCT 1560

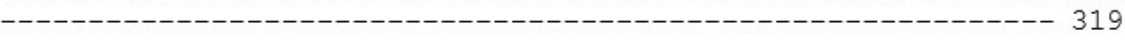

TCCACTGCTCGGAGCCGGGGTGGTTCAGGGTGTGCTTCGCCAACATGGACGACACGGCCA 1620

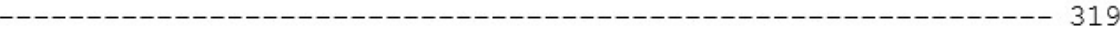

TGAAGATAGCGCTGAGGAGGATCGAGAGTTTCGTGTACCGGGAGAACGACGCCGCTGTGC 1680

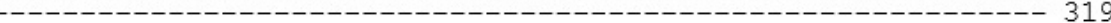

AGGCGAAGAACAAGAGGAGGTGGGACGAAGCGCTGCGGCTGAGCTTGCCTCGTCGGAGGT 1740 319

TCGAGGATCCGACCATCATGACACCACATCTGATGTCTCCCCACTCGCCTCTCGTTCAAG 1800

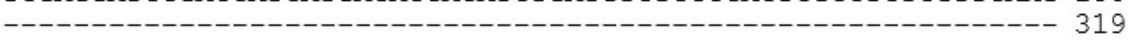

CCGCCACCTGAAACATCG 1818

319 
Supplemental Data 2. Alignment result of Musa acuminata AAA Group and pisang tongkat langit (Musa troglodytarum L.) ACO1 gene sequence. Starred sequences shows similar sequences (considered as conserved sequences).

ACO1_M.acuminata

ACO1_M.troglodytarum

ACO1_M.acuminata

ACo1_M.troglodytarum

ACO1_M.acuminata

ACO1_M.troglodytarum

ACO1 M.acuminata

ACO1_M.troglodytarum

ACO1 M. acuminata

ACO1_M.troglodytarum

ACo1_M. acuminata

ACO1_M.troglodytarum

ACO1_M.acuminata

ACO1_M.troglodytarum

ACo1_M.acuminata

ACO1_M.troglodytarum

ACO1 M. acuminata

ACO1_M.troglodytarum

ACO1 M. acuminata

ACO1_M.troglodytarum

ACO1_M.acuminata

ACO1_M.troglodytarum

ACo1_M.acuminata

ACO1_M.troglodytarum

ACO1_M.acuminata

ACO1_M.troglodytarum

ACO1_M.acuminata

ACO1_M.troglodytarum

ACO1_M.acuminata

ACO1_M.troglodytarum

ACo1 M.acuminata

ACO1_M.troglodytarum
ACGCGAGAGGTGGAAGCAAGGAGGGTGGAGAACCAGGCCAAAGGTGGTGGGGCTGAGAGA 60

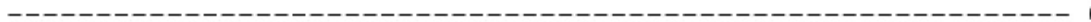

TGGCCAACTGGGTCACCCTATGGAATCGGCTCCGTTACGTCTTCCACTGCTGTTGCTCTC 120 0

GTCGATAGATCCTTCTCCAACTTTGCTTCCTCATTCATTTCGTCCCTCGACGTCAAGAAC 180

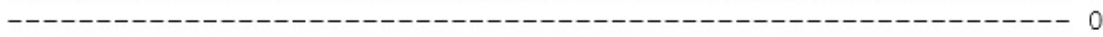

GCCTATAAATTGCCTGGTAATCAGCAGCACCTAGCACACTCCAGATAGAAAGCACAAGTG 240

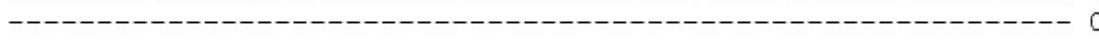

CAATCAGGGAAGAAAGAGCGTGTCATGGATTCCTTTCCGGTTATCGACATGGAGAAGCTT 300

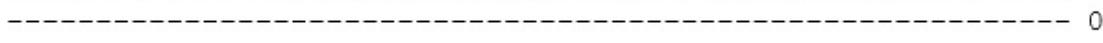

TTGGGAAGGGAGAGAGGAGCAGCCATGGAGATCCTCCGAGATGCTTGCGAGAAATGGGGC 360 GGAGAAATGGGGC 13 $* * * * * * * * * * * *$

TTCTTTGAGGGCTGAAGCATACATAACTGGTTTTGCTTCTTTGAACTATATATATTGCT 420 TCTTTGAGGTCCTGAAGCATACATAACTGGTTTTGCTTCTTTGAACTATACATACTGCT 73 $* * * * * * * * * * * * * * * * * * * * * * * * * * * * * * * * * * * * * * * * * * * * * * * * * * * * * * * * * *$

AAAAATGTACTATTTGCGCATGCAATCTGTGTGTAGATTTTAAACCATGGCATCTCACAT 480 --AAATGTACTATTTGCACATGCAATCTGTGCGTAGATTTTAAACCATGGCATCACACAT 131

$* * * * * * * * * * * * * * * \quad * * * * * * * * * * * * * * * * * * * * * * * * * * * * * * * * * * * * * * * * *$

ACCTCATGGATGAAGTGGAGAAGGTGAACAAAGAACAGTACAACAAATGCAGGGAGCAA 540 ACCTCATGGATGAAGTGGAGAAGGTGAACAAAGAGCAGTACGATAAAGGCAGGGAGCAA 191

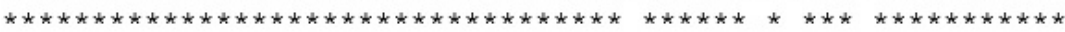

AAGTTCAACGAGTTCGCCAACAAAGCACTGGAAAACGCCGACTCAGAAATCGACCACCTC 600 AAGTTCAACGAGTTCGCCAACAAAGCACTGGAAAACGCCGACTCAGAAATCGACCACCTC 251

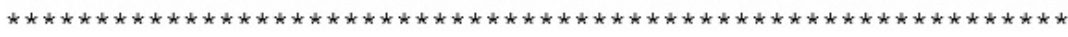

GACTGGGAAAGCACCTTTTTCCTGCGTCATCTCCCCGTCTCCAACATTTCTGAGATCCCC 660 GACTGGGAAAGCACCTTTTTCCTGCGTCATCTCCCCGTCTCCAACATTTCTGAGATCCCC 311 $* * * * * * * * * * * * * * * * * * * * * * * * * * * * * * * * * * * * * * * * * * * * * * * * * * * * * * * * * * * *$

GATCTTGATGACCAGTATAGGTTGCACGATCTGATCATGATGTCATCTTCTAGCCTTGTC 720 GATCTTGATGACCAGTATAGGTTGCACGATCTGATCATGATGTCATCTTCTAGCCTGGCC 371

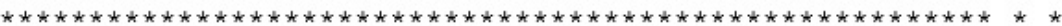

TTTTCACСTTGCTCATCGTTTCGTTTCTTGGGACGATGACTGCGTGCAGGAAGGCGATGA 780 TTTTCACCTTGCTCATCGTTTCGTTGCTTGGGACGATGACTGCGTGCAGAAAGGCGATGA 431

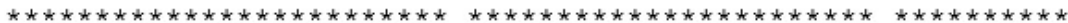

AGGAATTTGCTGCAGCGATAGAGAAGCTGGCAGAGCGGCTGCTCGACTTGCTGGGTGAGA 840 AGGAATTTGCTGCAAGGCCGGGAGACGCCAGGGGACGCCACCCGCCGAAA---------- 481 $* * * * * * * * * * * * * * * * * * * * *$

ACCTGGAGCTGGAGAAGGGGTACCTGAAGAAAGCCTTCTCTAATGGATCCAAGGGGCCAA 900

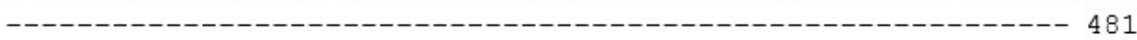

CCTTTGGGACCAAGGTCAGCAGCTACCCACCATGCCCACGCCCGGACCTGGTGAAGGGCC 960 
ACO1 M.acuminata ACO1_M.troglodytarum

ACO1 M. acuminata ACO1_M.troglodytarum

ACO1_M.acuminata

ACO1_M.troglodytarum

ACO1_M.acuminata

ACO1_M.troglodytarum

ACO1_M.acuminata

ACO1 1 M.troglodytarum

ACO1_M.acuminata

ACO1_M.troglodytarum

ACO1_M.acuminata

ACO1_M. troglodytarum

ACO1_M. acuminata

ACO1_M.troglodytarum

ACO1 M.acuminata

ACO1_M.troglodytarum

ACO1 M. acuminata

ACO1_M.troglodytarum

ACO1 M.acuminata

ACO1_M.troglodytarum
TGAGGGCGCACGCCGACGCCGGAGGCATCATCTTGCTCTTCCAGGACGACCAGGTCAGCG 1020 ------------------------------------------------------------- 481

GCCTGCAGTTCCTCAAGGACGGCGAGTGGCTGGACGTGCCCCCCATGCGCCATGCCATCG 1080

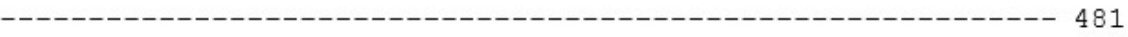

TCGTCAACCTCGGCGACCAGCTCGAGGTTTGGGTCCTCTTTGCTCTCGTTTCCGCTGCCC 1140

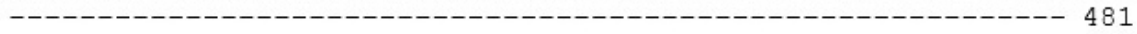

GTCGTCTGTGATGTTGAATGCAACGAGGTCTGCAGGTAATCACCAATGGCAAGTACAAGA 1200

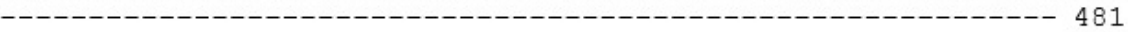

GCGTGGTGCACCGCGTGGTGGCTCAGACTGATGGCAACAGGATGTCGATTGCCTCCTTCT 1260

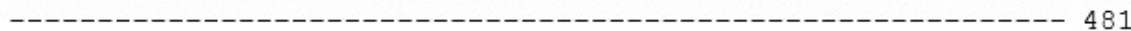

ACAACCCCGGGAGCGACGCTGTGATCTTCCCGGCCCCCGCTCTTGTGGAGAAGGAAGCGG 1320

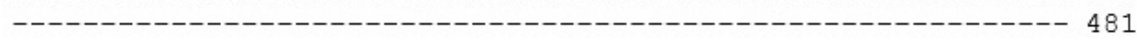

AGGAGAAGAAGGAGGTCTATCCGAGGTTCGTGTTCGAGGATTACATGAAGCTCTACGTCG 1380

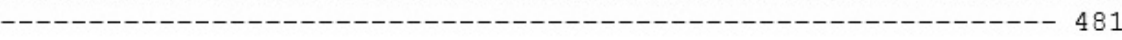

GGCATAAGTTCCAGGCCAAGGAGCCAAGATTCGAAGCCATGAAAGCCATGGAAGCAGTTG 1440

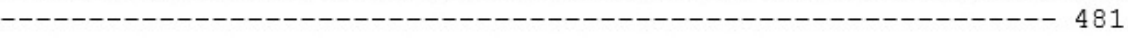

CCACCCACCCAATCGCTACCTCTTAAGTGACAGCCCCCAAGTTAGTGCATGTCGCTGTAC 1500

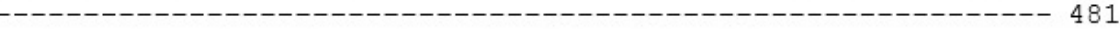

TTCGCGTTAGGAAGCTGTCGTCTATGTCTATGTAACCCGATGGAAGCGTGGTATGTACGT 1560

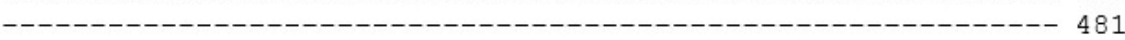

GTTTGAGCCTTTTCTAATGAAGCAAGTCATATA 1593

481 
Supplemental Data 3. Alignment result of DNA and cDNA sequence of pisang tongkat langit (Musa troglodytarum L.) ACS1. Black boxes are considered as exons.

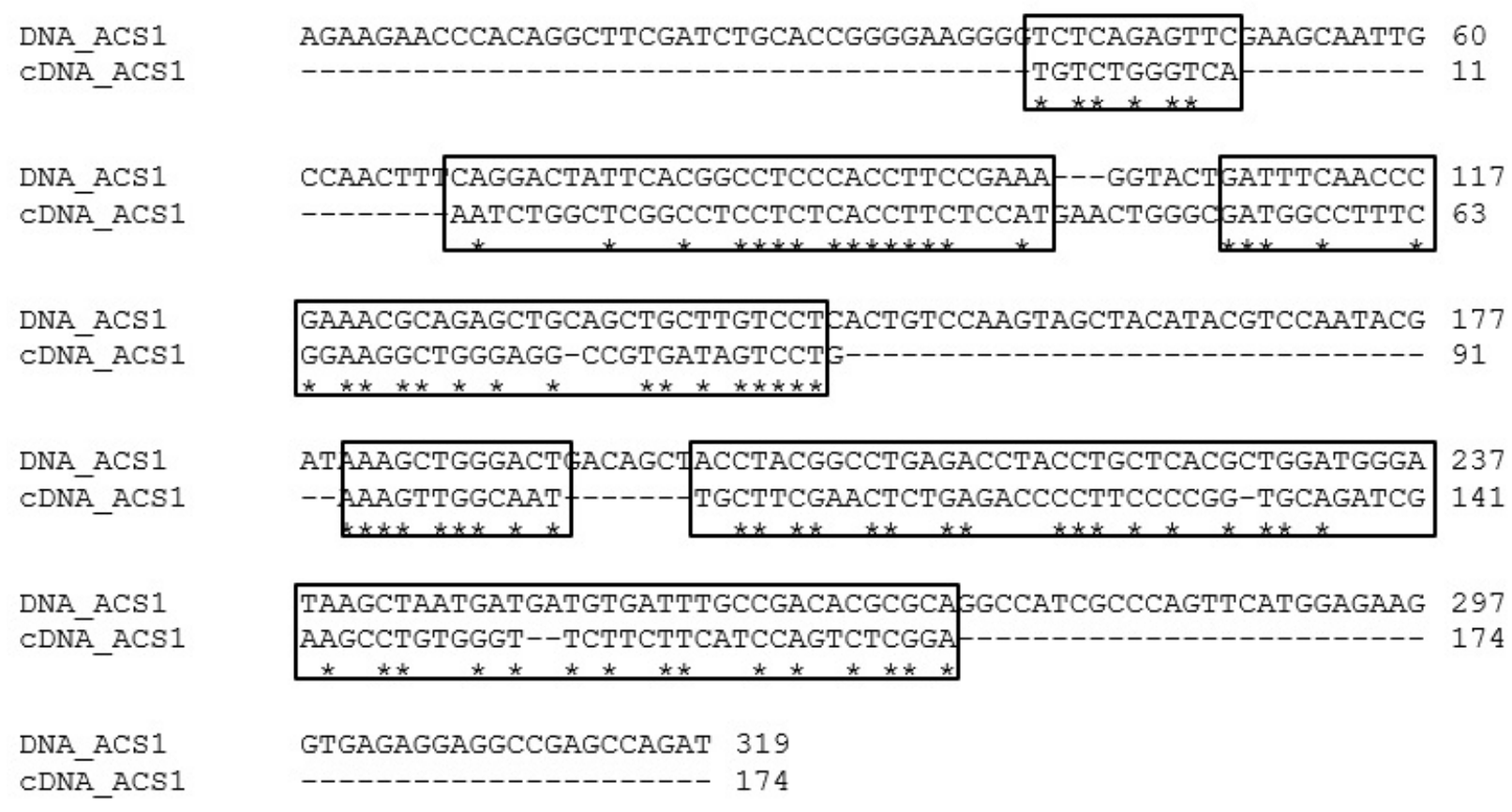


Supplemental Data 4. Alignment result of DNA and cDNA sequence of pisang tongkat langit (Musa troglodytarum L.) ACO1. Black boxes are considered as exons.

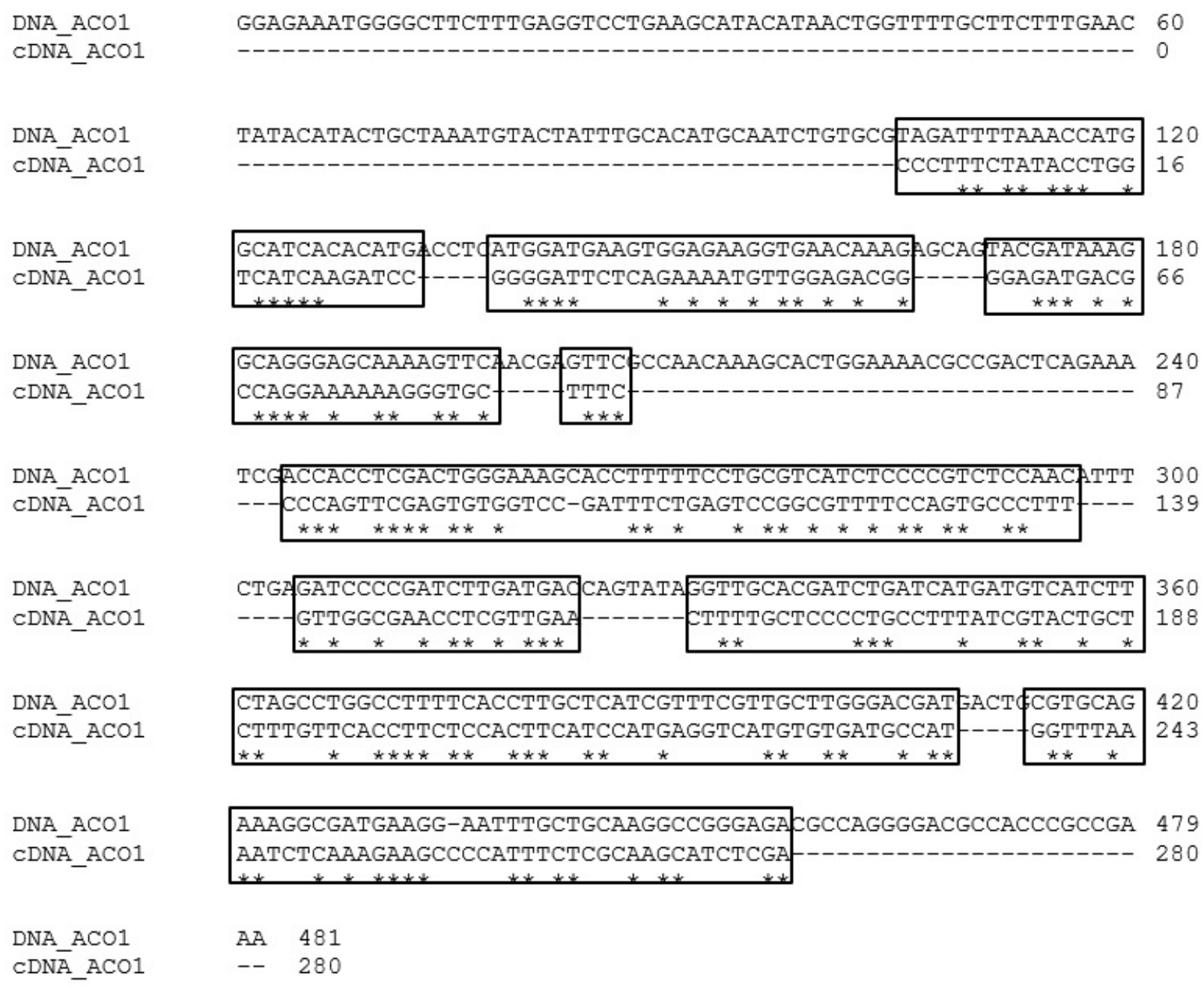

